\title{
Polarized Light Microscopy Coupled with Electron Backscatter Diffraction for 3D Serial Sectioning of Large Volumes of Twinned Titanium
}

\author{
Rodney McCabe ${ }^{1}$, Danel Savage ${ }^{2}$, Renuka Gogusetti ${ }^{2}$, Abhishek Bhattacharyya ${ }^{2}$ and \\ David Alexander IV ${ }^{2}$
}

\section{${ }^{1}$ Los Alamos National Laboratry, United States, ${ }^{2}$ Los Alamos National Laboratory, United States}

Twinning is an important deformation mechanism in hexagonal close packed metals such as titanium. The propensity of deformation twinning depends on microstructural characteristics such as grain orientation, grain size, and grain neighbors. Electron backscatter diffraction (EBSD) analysis from two-dimensional (2D) stereological sections has previously been used to effectively determine microstructural dependencies of twinning such as grain orientation and average grain size (Beyerlein, Capolungo, Marshall, McCabe, \& Tome, 2010; Capolungo, Marshall, McCabe, Beyerlein, \& Tome, 2009; Wronski et al., 2018). However, 3D microstructure characterization is necessary to fully define the effects of individual grain size and grain neighbor characteristics.

A common method for characterizing 3D microstructures is serial sectioning, which involves alternating sectioning and characterization steps. Sectioning processes typically involve mechanical polishing, femtosecond laser ablation, or focused ion beam milling. For the characterization steps, the crystallographic information available from EBSD is invaluable for understanding microstructural effects on twinning. However, EBSD data collection is normally the time-limiting step for generating large volume, high spatial resolution 3D EBSD data sets.

In the present work, we have developed a method for 3D microstructure characterization of twinned Ti that couples polarized light optical microscopy (PLM) and EBSD. The method maintains the critical crystallographic information inherent from EBSD but allows for large 3D data sets with minimized EBSD time. PLM is an effective method for imaging microstructures of materials with low crystal symmetry as different crystal orientations result in different light intensities. The primary challenge of quantitatively using PLM has been in effectively segmenting images into individual grains and/or twins. This difficulty is overcome by using three PLM images at different specimen rotations with unique region growing algorithms. PLM intensity varies with the c-axis inclination relative to the surface normal $(\propto)$ and with the azimuthal angle between the polarization direction and the crystal c-axis $(\theta)$ (Jin \& De Graef, 2020). For a single PLM image it is possible for grains with different $\alpha$ and $\theta$ to have similar intensities. However, imaging the sample at three different $\theta$ results in intensity differences between grains for at least one of the images as long as long as the c-axes of neighboring grains are not exactly parallel.

A sample of high purity rolled and recrystallized Ti with a strong basal texture and an average grain size of $30 \mu \mathrm{m}$ was compressed $2 \%$ in the direction parallel to the plate normal direction resulting in a high density of $<11-22>$ twins. We use mechanical serial sectioning by polishing with a 10:1 mixture of non-dry colloidal silica and $\mathrm{H}_{2} \mathrm{O}_{2}$ removing around $0.7 \mathrm{um}$ per slice. We perform PLM following every sectioning step to segment the microstructure into grains and twins for an area greater than $1 \mathrm{~mm} \times 1 \mathrm{~mm}$ with resolution approaching 200nm, and we perform EBSD after every 10-15 sectioning steps to determine the orientations of the segmented grains and twins. Three sets of $4 \times 5$ PLM montages at 200x magnification are obtained in a crosspolarization condition on a Zeiss Axiomat with sample rotations of $0^{\circ}, 22.5^{\circ}$, and $45^{\circ}$. The montages are then stitched and registered using the ImageJ/Fiji Grid/Collection stitching and ImageJ/Fiji Linear Stack Alignment with SIFT plugins, respectively (Fig. 1a-c). The three registered gray scale images are then converted into a single RGB color TIF (Fig 1d). Image slices are registered using the same registration plugin taking advantage 
of hardness indents around the periphery of the ROI. Individual slices are segmented into distinct grain and twin regions using appropriate region growing algorithms and image filters. Pixel RGB values are relatively constant at the interiors of grains and vary considerably for several pixels across grain and twin boundaries. Region growing seeds are chosen based on the eigenvalues of the RGB covariance matrix calculated based on the RGB values of the pixel and its $n^{\text {th }}$ nearest neighbors. Regions are grown by comparing the RGBs of neighboring pixels to the evolving average RGB of the growing region and determining whether their vector norm is less than some tolerance. The initial region growing with a small tolerance on the seed eigenvalues and RGB norm results in the interiors of large grains being well defined and grain and fine twin boundaries being thick and undefined (Fig. 1f). This initial region growing is followed by a dilation filter that allows the grains to grow further into the boundaries using a larger RGB vector norm tolerance (Fig. 1g). A second region growing with a larger RGB eigenvalue tolerance is used to define twins (Fig. 1h) and is followed by another dilation into undefined boundary or twin points with the neighbor sharing the most similar RGB (Fig 1h). The final step is to match the EBSD with the PLM data for slices in which both techniques are performed in order to assign orientations to PLM grains and twins. This involves converting the EBSD data (Fig. 2a) to an equivalent PLM image (Fig. 2b) and unwarping the EBSD spatial data to match the PLM spatial mapping.

At 500 EBSD frames per second, it takes nearly 3 hours to scan an area of $1 \mathrm{~mm}$ x $1 \mathrm{~mm}$ with a $0.5 \mu \mathrm{m}$ step size whereas it takes around 15 minutes to take the PLM images described above.

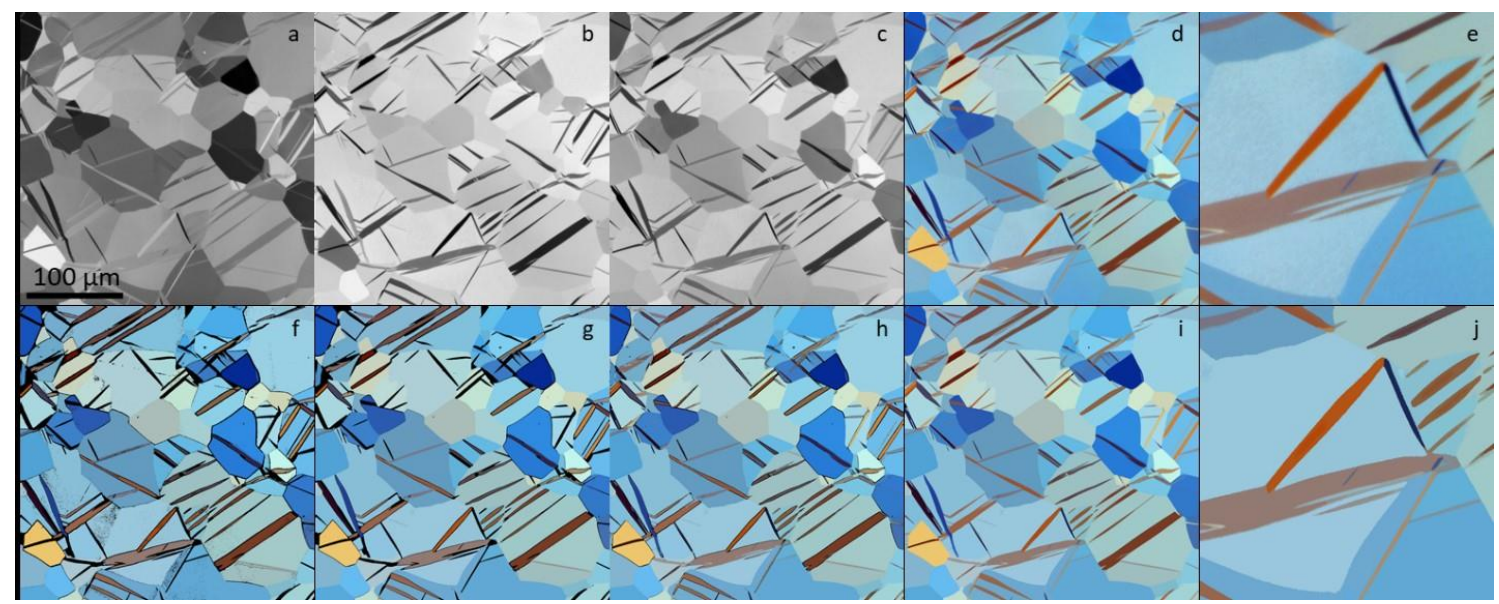

Figure 1. PLM images for sample rotations of a) $0^{\circ}$, b) $22.5^{\circ}$, and c) $45^{\circ}$ following stitching and registration. d) RGB from combined rotated images and e) zoomed in region from d). Segmentation consists of $\mathrm{f}$ ) region growing with a small tolerance on seed RGB eigenvalues, $g$ ) dilation, $f$ ) region growing with relaxed tolerances, and i) dilation. j) zoomed in segmented image.

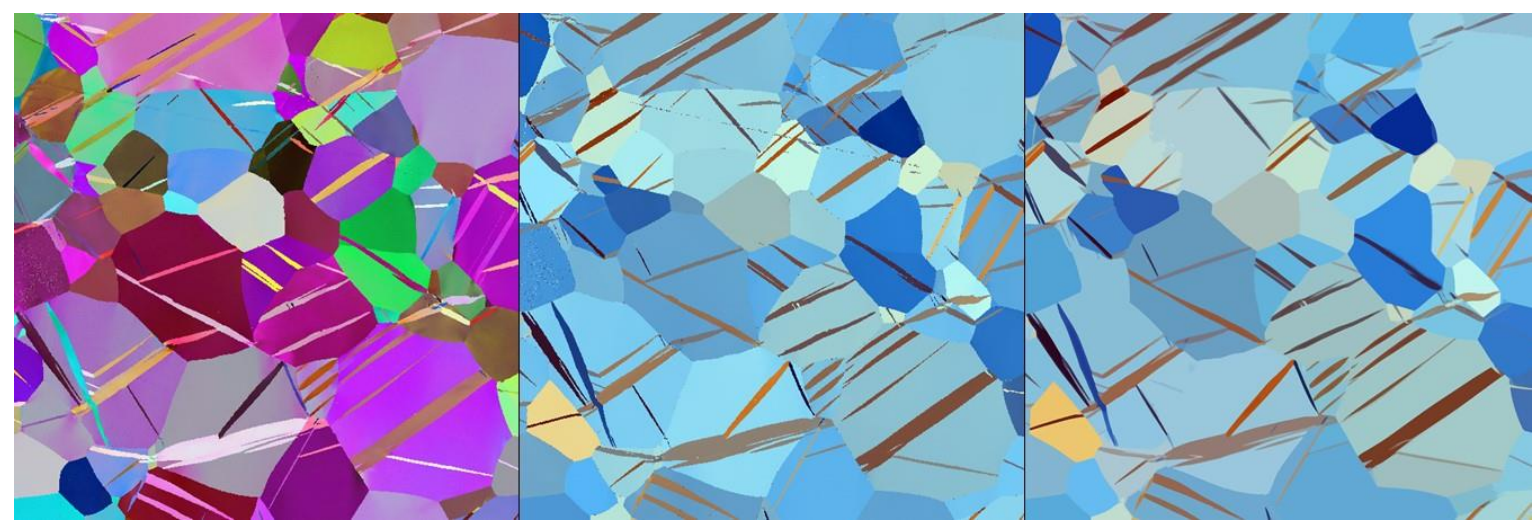


Figure 2. EBSD quaternion misorientation plot where pixels with different crystallographic orientations have distinct colors. b) EBSD data is converted to equivalent PLM colors using relationships between PLM intensity, $\alpha$, and $\theta$. c) Segmented PLM image for comparison.

References

Beyerlein, I. J., Capolungo, L., Marshall, P. E., McCabe, R. J., \& Tome, C. N. (2010). Statistical analyses of deformation twinning in magnesium (vol 90, pg 2161, 2010). Philosophical Magazine, 90(30), 40734074. doi:Pii 926495039

Capolungo, L., Marshall, P. E., McCabe, R. J., Beyerlein, I. J., \& Tome, C. N. (2009). Nucleation and growth of twins in Zr: A statistical study. Acta Materialia, 57(20), 6047-6056. doi:10.1016/j.actamat.2009.08.030

Jin, K.-W., \& De Graef, M. (2020). c-Axis orientation determination of alpha-titanium using Computational Polarized Light Microscopy. Materials Characterization, 167. doi:10.1016/j.matchar.2020.110503

Wronski, M., Arul Kumar, M., Capolungo, L., Madec, R., Wierzbanowski, K., \& Tome, C. N. (2018). Deformation behavior of CP-titanium: Experiment and Crystal plasticity modeling. Materials Science and Engineering a-Structural Materials Properties Microstructure and Processing, 724, 289-297. 